European Conference on Laboratory Astrophysics - ECLA

C. Stehlé, C. Joblin and L. d'Hendecourt (eds)

EAS Publications Series, 58 (2012) 161-165

www.eas.org

\title{
IN-LAB SHALLOW-WATER SIMULATIONS OF GASEOUS DISCS FOR ASTROPHYSICAL FLUID DYNAMICS
}

\author{
G.P. Chernikov ${ }^{1}$, A.M. Fridman ${ }^{2}$, E.N. Snezhkin ${ }^{1}$, J. Sommeria ${ }^{3}$ \\ and Yu.M. Torgashin ${ }^{2}$
}

\begin{abstract}
Presented is a brief review of firsthand laboratory experiments with the use of fast rotating shallow water for simulation of important 2D objects and phenomena of astrophysical fluid dynamics, which alternatively can only be studied theoretically, computationally and very rarely through astronomical observations. Among them are sheared supersonic rotation, instabilities of flows at such rotation, vortical solitary waves, turbulence, and wave over-reflection
\end{abstract}

We present below a general method for simulating supersonically and differentially rotating fluid by means of a free-surface liquid layer placed on a differentially rotating profiled bottom, with shallow-water waves spreading in the liquid like sound in a $2 \mathrm{D}$ gas. If the bottom is properly shaped, the layer can be made thin enough for the velocity of these surrogate "sonic waves", $\sqrt{g^{*} H}$, to be lower than the velocity of the global rotation, where $g^{*}$ is the norm of the vector sum of the gravity and centrifugal accelerations, and $H$ is the layer thickness. This gives a unique opportunity of working in vitro on scales larger than such characteristic lengths as Rossby and epicyclic radiuses (Nezlin \& Snezhkin 1993).

The method was first used in experiments (Antipov et al. 1981, 1985) on monopolar Rossby solitons, which are related to long-living large-scale eddies in planetary atmospheres and oceans as well as to drift solitons in magnetized plasmas (Fig. 1, top). Its broad physical adequacy became apparent from experimental results (Antipov et al. 1983) on Landau stabilization for counter flows with supersonic relative velocities (Fig. 1, bottom).

The next subject of the simulations (Morozov et al. 1984; Fridman et al. 1985; Nezlin et al. 1986, 1993) concerned a hydrodynamic mechanism that had been suggested as a generator of spiral structures in galaxies with a zone of sharp

\footnotetext{
${ }^{1}$ National Research Centre "Kurchatov Institute", 1 Acad. Kurchatova Sq., 123182 Moscow, Russia

2 Institute of Astronomy RAS, 48 Pyatnitskaya St., 119017 Moscow, Russia

3 Coriolis/LEGI, 21 avenue des Martyrs, 38000 Grenoble, France
} 

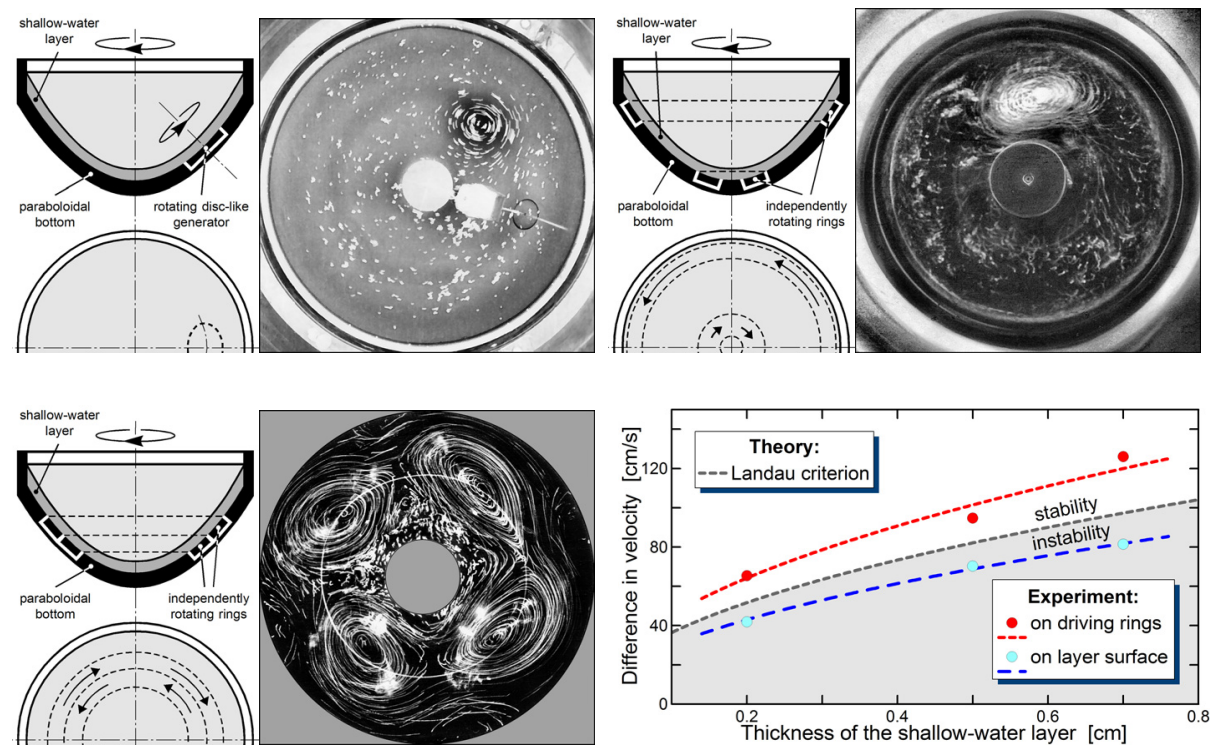

Fig. 1. Top: anticyclonic Rossby vortex drifting free and counter to the "global" rotation from the place of its impulse generation (left); Rossby autosoliton an anticyclone generated constantly by an anticyclonic shear between distant counter flows (right). Bottom: chain of cyclones generated by an instability of a cyclonic shear between adjacent counter flows and difference in velocities at the upper threshold of the instability of the cyclonic shear versus thickness of the layer (the Landau criterion for the threshold difference is presented as $\left.2 \sqrt{2 g^{*} H}\right)$.
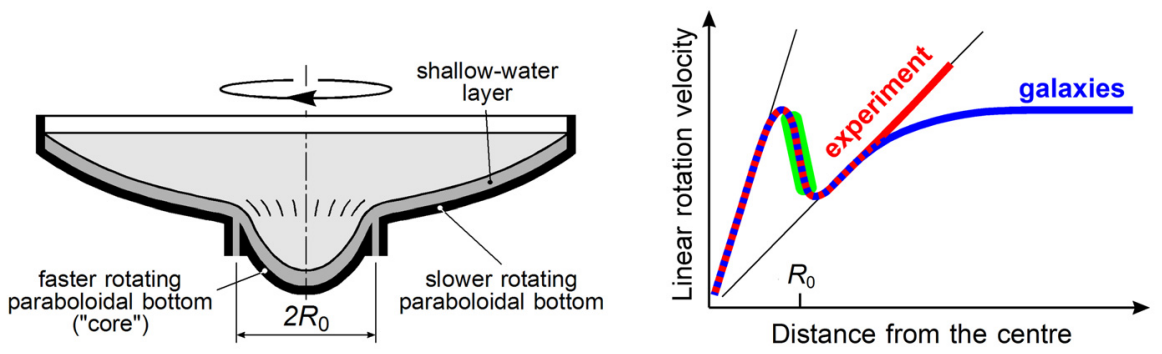

Fig. 2. Top: schematic of an experimental setup for simulating the conditions of gaseous discs of galaxies and a rotation curve with a sharp decrease in velocity.

decrease in their rotation curves. In this case, the bottom consisted of two coaxial rigid parts, the inner rotating faster than the outer one so that the layer simulated the gaseous disc typical of such galaxies (Fig. 2). 

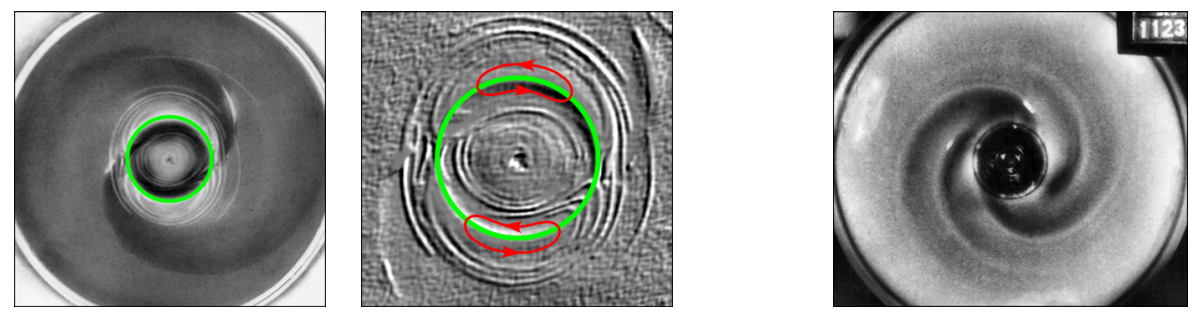

Fig. 3. Top: left: interarm anticyclonic vortices in the zone of the generating shear on the corotation radius, forming a single whole with the spiral waves. Right: branching of arms in an intermediate spiral structure at a stage of the transition from mode four to mode two caused by a slow increase in rotation velocity of the core.

The anticyclonic shear in the layer experienced centrifugal (CI) and/or Kelvin-Helmholtz (KHI) instabilities with generation of trailing spiral waves on the surface of the liquid. The experimental anticyclonic vortices, which were found on the corotation radius between the spirals, should have analogues existing in spiral galaxies of this kind (Fig. 3, left). This prediction came true with the discovery of natural anticyclones in purposive observations performed for a number of galaxies (Fridman 2007). The experimental simulations showed that the branching and asymmetry of the spiral arms in galaxies could be a result of transitions from one mode of the instability to another (Fig. 3, right).

The reverse configuration (Fig. 4, left), with cyclonic and sufficiently "supersonic" difference in the velocities of the bottom parts, was used in our search (Fridman et al. 2008) for the over-reflection instability that had been repetitively discussed in relation to turbulence in astrophysical discs (Fridman et al. 2009). It is due to this instability that the annular shear can come unstable - if the two instabilities above (CI and $\mathrm{KHI}$ ) vanish like they do in this configuration. In the simulations, we succeeded in revealing this elusive instability that actually had never been observed before. Its behaviour in the experiments ascertained to be in close agreement with theoretical predictions (Fig. 4, left). It is possible to interpret the wave structures generated by this instability as resonant superposition of Huygens-Mach fronts that, after having been induced by a supersonically revolving seed disturbance, are multiply over-reflected from the shear (Fig. 4, right).

The advanced scheme of the experimental simulation involves an MHD technique (Fig. 5, left): a relatively thick bed of mercury plays a role of a self-adjusting bottom, with a layer of working liquid above and a number of coaxial ring electrodes underneath. As a variable voltage is imposed across the electrodes, a variable distribution of radial electric current $I(r)$ is set in mercury. When vertical magnetic field $B$ is applied to such a system, a variable radial distribution of rotation velocity $\Omega(r)$ is set in the bed. The surface of the bed automatically arranges itself into an equilibrium shape corresponding to the rotation. The efficacy of the scheme was verified in experiments with an unequalled setup built in ENS de Lyon (France) in frame of a French-Russian research project. The setup allowed 

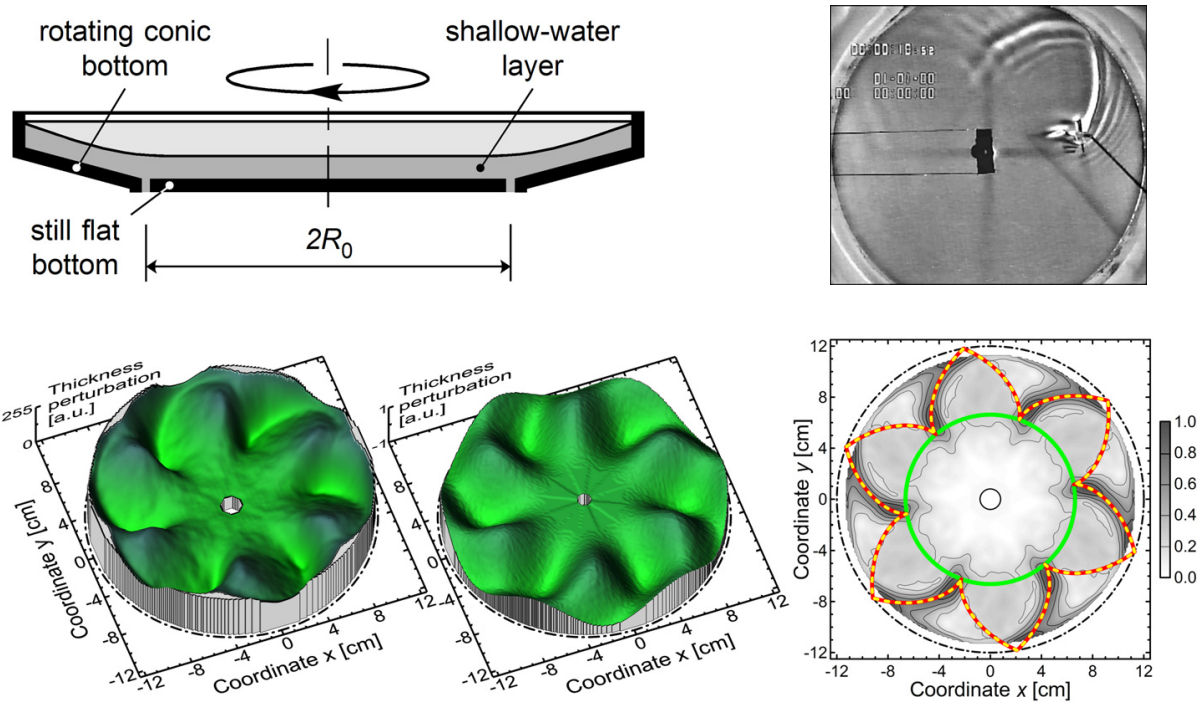

Fig. 4. Left: schematic of the experimental setup for studying the over-reflection instability and thickness perturbation in unstable mode six as experimental (below left) and theoretical (below right) results. Right: waves from a body revolving in the layer with their reflections from a circular wall and magnitude of the thickness gradient in the wave structure (experiment) overlaid with a resonantly multi-reflected shock front (theory).
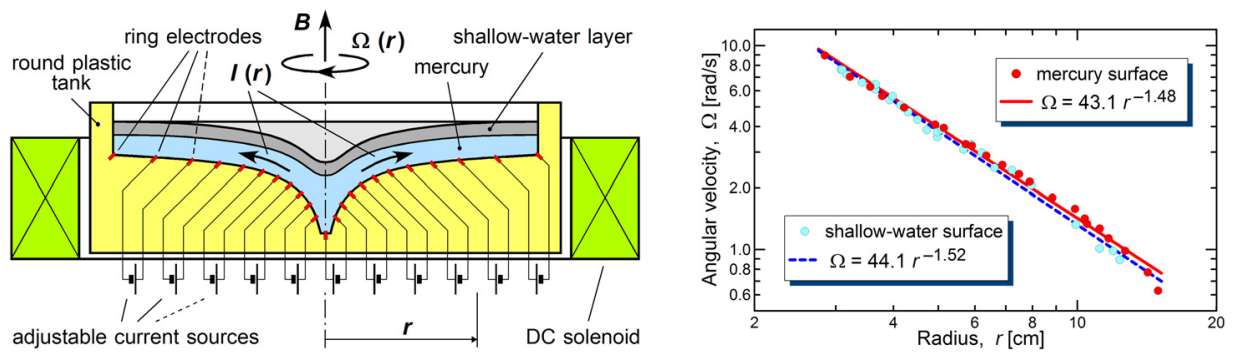

Fig. 5. Left: schematic of a setup with smooth differential rotation of a shallowwater layer by means of underlying MHD-driven mercury. Right: Keplerian rotation realised in shallow water over the MHD-driven mercury 'bottom' (shallow-water layer is about $0.5 \mathrm{~cm}$ thick).

simulating in a broad range of smooth supersonic rotation profiles including the Keplerian (Fig. 5, right).

E.N.S. and Yu.M.T. acknowledge Russian Foundation for Basic Research for partial support of their work on this review in frame of RFBR Project 11-02-01248a. 


\section{References}

Antipov, S.V., Nezlin, M.V., Snezhkin, E.N., \& Trubnikov, A.S., 1981, JETP Lett., 33, 351

Antipov, S.V., Nezlin, M.V., Rodionov, V.K., Snezhkin, E.N., \& Trubnikov, A.S., 1983, JETP Lett., 37, 378

Antipov, S.V., Nezlin, M.V., Snezhkin, E.N., \& Trubnikov, A.S., 1985, Sov. Phys. JETP, 62, 1097

Fridman, A M., Morozov, A.G., Nezlin, M.V., \& Snezhkin, E.N., 1985, Phys. Lett. A, 109, 228

Fridman, A.M., 2007, Sov. Phys., 50, 115

Fridman, A.M., Snezhkin, E.N., Chernikov, G.P., et al., 2008, Phys. Lett. A, 372, 4822

Fridman, A.M., Bisikalo, D.V., Boyarchuk, A.A., Torgashin, Yu. M., \& Pustil'nik, L.A., 2009, Astron. Rep., 53, 750

Morozov, A.G., Nezlin, M.V., Snezhkin, E.N., \& Fridman, A.M., 1984, JETP Lett., 39, 613

Morozov, A.G., Nezlin, M.V., Snezhkin, E.N., \& Fridman, A.M., 1986, Sov. Astron. Lett., 12, 213

Nezlin, M.V., Snezhkin, E.N., 1993, Rossby Vortices, Spiral Structures, Solitons (Springer, Berlin) 